\title{
IMPLEMENTACIÓN DE UNA ACTIVIDAD EDUCATIVA EN MODO COLABORATIVO CON APOYO DE SMARTPHONES: UNA EXPERIENCIA UNIVERSITARIA
}

\author{
IMPLEMENTATION OF AN EDUCATIONAL COLLABORATIVE ACTIVITY \\ WITH SMARTPHONES SUPPORT: A UNIVERSITY EXPERIENCE
}

\author{
Javier Organista-Sandoval; javor@uabc.edu.mx \\ Instituto de Investigación y Desarrollo Educativo, UABC. \\ Arturo Serrano-Santoyo; serrano@cicese.mx \\ Centro de Inv. Científica y de Enseñanza Superior de Ensenada (CICESE).
}

\begin{abstract}
RESUMEN
El sector educativo se ha convertido en un campo fértil para explorar el potencial de aplicación de los dispositivos portátiles como apoyo al aprendizaje. El objetivo de este artículo es presentar los resultados de la implementación de una actividad educativa en modo colaborativo mediante el uso de smartphones. El artículo identifica los elementos clave que apoyan la interacción y comunicación ubicua de grupos de estudiantes universitarios en un contexto que requiere de un intenso nivel de interacción para la entrega de tareas a corto plazo.
\end{abstract}

PALABRAS CLAVE: aprendizaje móvil; uso pedagógico de smartphones

\begin{abstract}
The educational sector has become fertile ground for exploring the potential application of mobile handheld devices to support learning. The aim of this paper is to present the results of the implementation of an educational activity in a collaborative way using smartphones. This article identifies the key elements that support ubiquitous communication and interaction of groups of university students in a context that requires an intense level of interaction for the delivery of short-term assignments.
\end{abstract}


KEYWORDS: $m$-Learning; educational use of smartphones.

\section{INTRODUCCIÓN}

El crecimiento explosivo de las comunicaciones inalámbricas a nivel mundial, en particular de la telefonía celular, ha permitido la penetración de los llamados dispositivos portátiles en prácticamente todas las áreas del quehacer humano; así mismo, el número de usuarios de Internet muestra cifras cada vez en aumento (Mansfield, 2011). El sector educativo no ha estado aislado de estos dos fenómenos convergentes y en especial, desde la década pasada se han desarrollado un gran número de experiencias y proyectos de aplicación con dispositivos portátiles y acceso a Internet en los diferentes niveles educativos y en entornos rurales, suburbanos y urbanos. La posibilidad de acceder a fuentes de información, comunicación e interacción en forma ubicua proporciona un amplio potencial pedagógico. A su vez, el despliegue de redes inalámbricas de última generación ( $3 G$ y $4 G$ ) y la disponibilidad comercial de dispositivos con grandes capacidades de procesamiento y conectividad a Internet han creado un entorno favorable para explorar el uso educativo de tales dispositivos.

Las capacidades tecnológicas avanzadas incorporadas en los dispositivos portátiles denominados smartphones y el desarrollo de contenidos con énfasis educativo se han convertido en elementos clave en el llamado m-learning, disciplina que ha sido motivo de atención para buscar por un lado, aumentar la cobertura, calidad y eficiencia de los servicios educativos y por otro alternativas de aprendizaje fuera del aula (Berger, Mohr, Nösekabel \& Schäfer ,2003; Brown, 2009). El m-learning es considerado, en la visión de los autores, como el conjunto de procesos y tecnologías de apoyo al aprendizaje mediante terminales portátiles. No solo es el concepto de explorar maneras de apoyar el aprendizaje en forma nómada o ubicua el que se involucra en esta visión, los aspectos de interacción, interactividad, inmediatez, intención de uso y otros de carácter ergonómico, de usabilidad y adopción forman parte de tal enfoque, el cual pretende abordar de manera integral el despliegue y aplicación de los smartphones en el contexto educativo (Serrano-Santoyo y Organista-Sandoval, 2010).

En el presente documento se describe la experiencia, desde una perspectiva exploratoria, alrededor de la noción de colaboración y comunicación de un grupo de estudiantes universitarios que tuvieron el objetivo específico de desarrollar una tarea que requirió de comunicación e interacción intensas para su cumplimiento y entrega en una fecha específica. El diseño de esta experiencia se centró en la resolución de una asignación concreta: la elaboración de un ensayo sobre m-learning en forma colaborativa utilizando tres elementos clave (Kantel, Tovar y Serrano, 2010):

- Smartphones con capacidades de acceso a Internet y conectividad 3G/4G;

- el desarrollo de un Entorno de Colaboración Móvil (ECM) y

- el desarrollo de una herramienta de colaboración con interfaz sencilla que facilite la interacción entre los estudiantes.

A la par de la descripción de la experiencia, se señalan las principales bondades y limitaciones derivadas de su implementación con un grupo de estudiantes universitarios. 


\section{REFERENTES TEÓRICOS}

Las capacidades tecnológicas de comunicación, procesamiento, conectividad a Internet y otros atributos como proximidad física e inmediatez de uso han creado interés de investigadores, educadores y profesionales para aprovechar los dispositivos portátiles tipo smartphone como herramienta pedagógica. El smartphone -visto como evolución de los teléfonos celularessoporta aplicaciones convergentes que lo convierten en un elemento versátil con potencial de contribución a las distintas modalidades educativas (presencial, en línea, a distancia, entre otras). Si bien existe una amplia gama de contribuciones que exploran el potencial de los smartphones en el contexto educativo (Kukulska-Hulme, 2007; Sharples, 2005) se observa la necesidad de fortalecer el marco conceptual del m-learning mediante investigaciones que incorporen aspectos cognitivos y tecnológicos, de conectividad del dispositivo, de movilidad (espacial, temporal y contextual) y de análisis contextual del ejercicio educativo en los espacios formales e informales, por mencionar algunos.

Es importante considerar que el atributo de movilidad, tanto del dispositivo como de la persona, tiene una nueva connotación cuando se dispone de los servicios de información y comunicación en todo momento y todo lugar. La posibilidad de disponer de un dispositivo con funciones de comunicación de datos y de procesamiento que cabe en la bolsa de la camisa o en la palma de la mano puede propiciar situaciones de aprendizaje con características particulares (KukulskaHulme, 2007; Traxler, 2007). En tales circunstancias, un punto de partida en los referentes teóricos es la definición misma de aprendizaje móvil o m-learning, la cual debe ir más allá de la visión tecno-céntrica que lo concibe como aquella situación de aprendizaje asistida con dispositivos portátiles.

En términos de lo cambiante de esta tecnología y su acelerada penetración en la sociedad, se detecta un vacío de sustento teórico alrededor del m-learning (Corbeil y Valdes-Corbeil, 2007). Ante dicha carencia, es común recurrir a teorías de aprendizaje como conductismo, constructivismo, aprendizaje situado, informal/ a lo largo de la vida o como apoyo al proceso enseñanza-aprendizaje (Naismith, Lonsdale, Vavoula y Sharples, 2009). Parte del problema es precisamente que se recurre a desarrollos teóricos que fueron concebidos para un proceso educativo presencial, o en el mejor de los casos para modalidades híbridas o a distancia. En muchas ocasiones, estas aportaciones se quedan en el plano meramente teórico por lo que las estrategias prácticas dependerán de la interpretación que le dé el investigador o docente.

Una propuesta interesante en torno al m-learning es hecha por Koole (2009), quien visualiza al m-learning como un proceso que resulta de la convergencia de: i) las tecnologías móviles, ii) las capacidades del aprendizaje-humano y de las iii) interacciones sociales. La idea tras el modelo propuesto (FRAME: Framework for the Rational Analysis of Mobile Education) es contribuir y orientar el desarrollo futuro de materiales educativos y especificaciones en torno a estrategias didácticas y de aprendizaje para entornos móviles (Koole \& Ally, 2009).

De acuerdo con Fife y Pereira (2005), las aproximaciones teóricas actuales en torno al $\mathrm{m}$ learning se basan principalmente en aspectos relacionados con la comunicación y los contenidos (cuerpos de información). Por un lado, la comunicación posibilita que un estudiante establezca contacto inmediato o diferido con otros estudiantes y/o asesores/maestros a la vez 
que propicia la eventual conformación e integración de redes sociales. Estas acciones se ubican en el llamado constructivismo social. Respecto a los contenidos desde la perspectiva móvil, Brown (2005) destaca la importancia de la navegación entre los distintos contenidos de la red Internet como una forma fundamental para propiciar la adquisición del conocimiento. A esta tendencia o aproximación teórica, dicho autor la denomina navigationism (navegacionismo).

En la misma dirección, una clasificación interesante del aprendizaje asistido con dispositivos portatiles fue propuesta por Naismith, Lonsdale, Vavoula \& Sharples (2009). Para estos autores, dicho aprendizaje puede ser: i) didáctico, entendido este al que recurre a material educativo que se accede desde un dispositivo portátil. Se incluyen e-libros e información de la Web. Indudablemente con las capacidades y limitaciones inherentes a tales dispositivos. Por otro lado se tiene al ii) aprendizaje discursivo, el cual se basa en el desarrollo de interacciones y prácticas discursivas entre comunidades de m-aprendices. Desde la perspectiva descrita en (Sharples, Taylor \& Vavoula, 2009), la aproximación conversacional, con maestros, estudiantes o inclusive con nosotros mismos, es fundamental para el m-learning. Si bien la conversación es un recurso pedagógico importante bajo cualquier modalidad educativa -presencial, a distancia, online 0 hibrida- bajo la óptica portátil, es particularmente importante ya que se dispone de la posibilidad de comunicación en todo momento y lugar.

Con base en lo antes expuesto, en el presente documento se describe la implementación de una actividad educativa grupal, utilizando como elemento mediador el dispositivo smartphone y con la disposición de un entorno de colaboración móvil, puesto a disposición de los participantes desde un servidor web. Documentar una experiencia de este tipo, reviste particular importancia ya que da cuenta de lo que implica: desarrollar recursos educativos para su acceso desde smartphones, las bondades y limitaciones del uso de estos dispositivos y las implicaciones pedagógicas de este tipo de intervención.

\section{MÉTODO}

El método se enfoca a describir la experiencia de realización de una actividad grupal con propósito educativo (m.Actividad) con apoyo de un Espacio de Colaboración Móvil (ECM) alojado en un servidor web, donde cada uno de los participantes de la investigación podía acceder desde su dispositivo portátil (ver fig. 1). Bajo esta idea, en el presente apartado se presenta primeramente la información concerniente al desarrollo del ECM, a las características funcionales de los smartphones y a la descripción de la m.Actividad. En un segundo momento, se describe la intervención para realizar dicha actividad. 


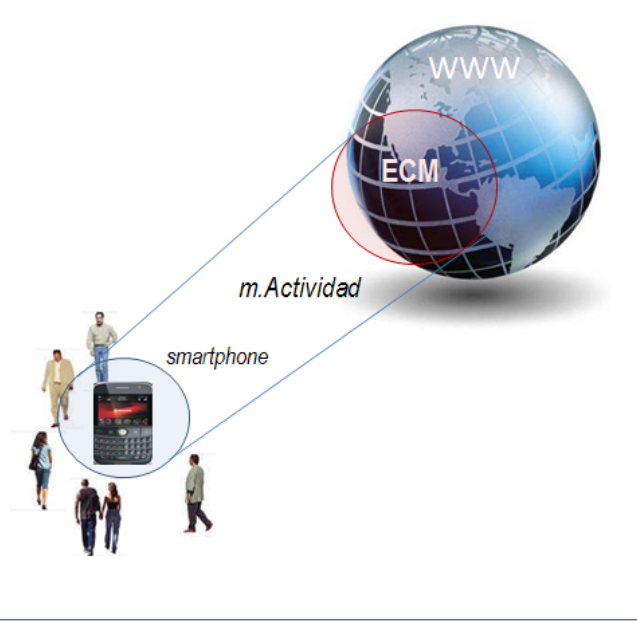

Fig. 1. Elementos de la experiencia colaborativa con apoyo de smartphones

\subsection{Descripción del Espacio de Colaboración Móvil}

EI ECM es una herramienta de colaboración alojada en un servidor web ( http://m.educa.ens.uabc.mx/ ), que considera los cambios culturales en el proceso educativo derivados de la convergencia de las tecnologías móviles y el cómputo ubicuo. Parte de la premisa de que los jóvenes universitarios muestran una tendencia y disposición natural al uso de tecnología en su proceso de aprendizaje; nativos digitales, desde la visión de Prensky (2001). Así, el diseño del ECM se centra en aprovechar la familiarización e inclinación de los estudiantes al uso de redes sociales en su interacción social y extrapolar esas experiencias a un contexto educacional. El objetivo del ECM es apoyar a grupos estudiantiles universitarios en la realización de tareas que requieran de interacción y comunicación entre ellos. El diseño contempla proporcionar un espacio virtual de colaboración y comunicación para su acceso mediante dispositivos portátiles 3G/4G (smartphones).

Conformación de los grupos de trabajo. El ECM contempla alojar las actividades colaborativas de diferentes grupos estudiantiles en forma simultánea. Se estima un máximo de cinco participantes por grupo, esto para facilitar el flujo y despliegue de información visual con los smartphones. La formación de los grupos se realiza de forma presencial, al inicio de los trabajos, y precisamente el facilitador del curso define los temas y acuerda la fecha de entrega. A su vez, cada grupo nombra a su respectivo líder de proyecto, cuya labor consiste en coordinar y monitorear los avances de su grupo.

Al inicio de las actividades, cada miembro del grupo ingresa al ECM y registra sus datos. Con base en este registro, la dinámica de interacción móvil y ubicua de los participantes queda habilitada y el desarrollo de las actividades de colaboración para el cumplimiento de las metas establecidas dependerá de los factores socioculturales, tecnológicos, de usabilidad y de su interrelación (Álvarez y Schacther, 2006).

Descripción de los módulos del ECM. El ECM está conformado por siete módulos (véase fig. 2). El primero, denominado Progreso, proporciona información acerca de la tarea o proyecto por realizar. El siguiente modulo, Avisos, muestra a los integrantes del grupo información relevante relacionada con el desarrollo de la actividad por realizar. Los avisos deben ser mensajes cortos 
para su adecuada visualización en la pantalla reducida de los dispositivos smartphones. El tercer módulo denominado Repositorio contiene información de apoyo (texto, imágenes, multimedios) para el desarrollo de la actividad o proyecto. Cada grupo configura su repositorio de forma independiente y para su uso exclusivo. El modulo siguiente, denominado Chat, permite la comunicación por medios electrónicos en tiempo real. El quinto módulo, Convergencia, es una Wiki donde a través de una página web, un mismo documento puede ser editado por los miembros del grupo. Esto propicia la construcción colaborativa de documentos y los acuerdos entre los participantes. El módulo siguiente, denominado Contacto, contiene los perfiles e identificación necesaria para localizar a los miembros de cada grupo. El último módulo, Ayuda, contiene el manual de usuario del ECM y algunas respuestas a preguntas frecuentes.

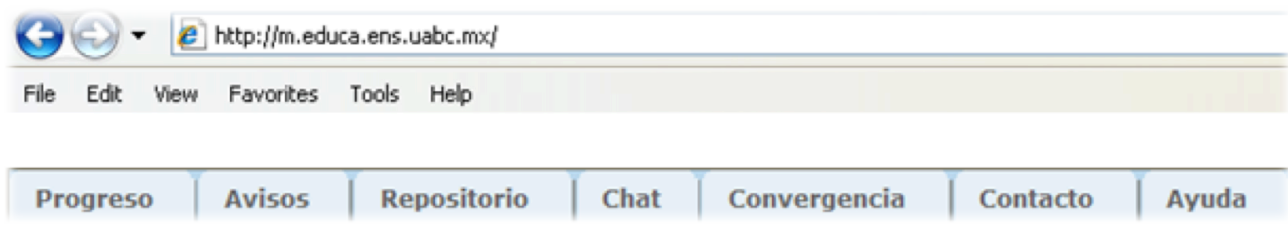

Fig. 2. Módulos del ECM

Desarrollo del ECM. Para la conformación del ECM se consideraron tres aspectos claves. El primero fue permitir la interacción y comunicación de los estudiantes mediante algún dispositivo smartphone independientemente del tipo o modelo, sistema operativo y navegador. El segundo aspecto fue utilizar la conectividad que ofrece la tecnología celular que le permite al usuario obtener una conexión a Internet de forma rápida y transparente. El tercer aspecto clave del ECM es la arquitectura del servidor web que integra los servicios de almacenamiento de materiales de apoyo al aprendizaje y las bases de datos que lo conforman. Esta arquitectura permite la operación y acceso al ECM sin necesidad de desarrollar aplicaciones específicas para cada tipo y modelo de smartphone.

Para lograr una buena experiencia de usuario en la colaboración, se deben considerar las capacidades y limitaciones de los smartphones. Es fundamental desarrollar aplicaciones que consideren la limitación del tamaño de pantalla y la capacidad de almacenamiento y procesamiento de los dispositivos móviles. El diseño de las pantallas y la definición de contenidos son cruciales para que la interacción colaborativa entre los estudiantes sea al menor costo posible de acuerdo a los planes tarifarios y esquemas de pago de proveedores de telefonía celular.

\subsection{Descripción de los dispositivos portátiles utilizados}

Como punto de partida, se identificaron las características principales, aspectos de conectividad, aplicaciones y funcionalidad de los seis dispositivos smartphones utilizados. En la tabla 1 se presentan las dimensiones consideradas y las variables consideradas. 


\begin{tabular}{ll}
\hline \multicolumn{1}{c}{ Dimensión } & \multicolumn{1}{c}{ variables } \\
\hline Características & Velocidad de procesamiento \\
& Pantalla sensible \\
& Resolución de la pantalla \\
& Tipo/tamaño de letra \\
& Peso y tamaño del celular \\
& Duración de batería \\
& Multitareas \\
& Calidad de cámara video/audio \\
& Costo \\
Conectividad & Acceso a Internet \\
& Email \\
& Conexión a PC \\
& Descargas \\
& Media player \\
Aplicaciones & Manejo de contactos \\
& Calendario/agenda/alarma \\
& Word, Excel y PowerPoint \\
& Edición de texto \\
& Organización de archivos \\
& Visibilidad de textos en \\
& espacios abiertos \\
Facilidad de manejo
\end{tabular}

TABLA 1. Aspectos básicos considerados para la caracterización de los smartphones

A partir de las dimensiones seleccionadas y las variables especificadas se elaboró el cuestionario de evaluación de la funcionalidad de los smartphones. Para ello, se consideraron 24 reactivos con cinco opciones de respuesta: no aplica, pésimo/a, regular, bueno/a y excelente (Codificación 0-4). El cuestionario completo se muestra en el anexo A. Respecto al sistema operativo de los seis equipos utilizados, dos de ellos contaban con el sistema operativo Windows Mobile 6.0 professional; otros dos smartphones operaban bajo Symbian $\mathrm{S} 60$ y los dos restantes con I-phone OS. Sin pretender ser un comparativo entre dispositivos, el objetivo se dirigió a obtener una caracterización funcional de los equipos que sirvieron como punto de conexión y enlace entre los participantes del grupo de estudio.

\subsection{Descripción de la m.actividad}

La actividad central consistió en elaborar un ensayo en formato digital, que de manera fluida y coherente aborde la definición misma de smartphone, sus características y potencial pedagógico. Su elaboración requirió de la participación colaborativa del equipo, quienes con un lenguaje sencillo, alejado de tecnicismos, en la medida de lo posible, reflexionaron en torno al uso educativo de esta tecnología. Para la elaboración de esta actividad, se utilizó el ECM con accesos desde cada smartphone de los miembros del equipo. A continuación se presenta el documento central de la actividad: 
m.Actividad. Indudablemente, el potencial pedagógico de las tecnologías móviles es enorme. Por mencionar algunos ejemplos, el estudiante puede localizar información en diversos formatos, establecer comunicación con expertos o con sus compañeros, intercambiar información, entre muchas otras actividades. Sin embargo, también se ve expuesto a distractores como juegos, películas, uso informal de redes sociales, entre otros. En algunos casos, existen estudiantes que desconocen las capacidades y usos de los llamados teléfonos inteligentes o smartphones o inclusive la existencia misma de estos dispositivos. Ante estos problemas, se considera necesario la elaboración de un ensayo, en formato digital y con apoyo multimedia deseable, que dé cuenta de las bondades y limitaciones de los smartphones y que a su vez, sirva como un documento orientador hacia la comunidad estudiantil en torno a esta temática.

\subsection{Aplicación}

\section{Participantes}

El estudio se realizó durante el primer semestre de 2010. El grupo colaborativo se conformó con 6 integrantes (dos investigadores y cuatro estudiantes de posgrado). La idea fue integrar un grupo cuyos participantes tuvieran igualdad de roles para la realización de la tarea ( $m$.Actividad ). Por acuerdo del grupo, se designó a uno de los estudiantes como líder de proyecto.

\section{Instrumentos}

Guía del grupo focal. Tuvo el propósito de recopilar información en torno a: i) aspectos técnicos del servidor que alojó al espacio de colaboración móvil; ii) desarrollo del ECM; iii) opinión acerca del smartphone como punto de acceso al servidor; iv) opinión acerca de la m.Actividad y finalmente, v) las ventajas y desventajas de la tecnología portátil. Los participantes del grupo focal fueron los seis miembros del grupo colaborativo de investigación.

Bitácora de la actividad con el ECM. Se llevó un registro de la actividad en el ECM, así como de los avances a la tarea grupal considerando las aportaciones hechas en cada uno de los espacios de colaboración del servidor (ECM).

\subsection{Procedimiento}

De manera inicial, los participantes del grupo se reunieron para conocer la información en torno a la actividad y los acuerdos para su elaboración. Asimismo, se designó a un coordinador del grupo. La m.Actividad se colocó en el ECM y se dispusieron de 10 días hábiles para su entrega y publicación en el mismo ECM.

\subsection{Análisis de resultados}

Para el análisis estadístico cuantitativo de los datos se utilizó el programa SPSS ${ }^{\circledR}$ versión 17.0. Se estimaron los descriptivos básicos para las variables consideradas en el cuestionario de evaluación de la funcionalidad de los smartphones. Para conocer la opinión de los participantes en torno al funcionamiento del ECM y al desarrollo de la actividad, se realizaron las 
transcripciones a los archivos con audio del grupo focal. A partir del texto obtenido, se identificaron las ideas principales y se relacionaron según el módulo de interés del ECM.

\section{RESULTADOS}

\subsection{De los smartphones utilizados}

De la escala utilizada (0-4) en el cuestionario se consideró su equivalente porcentual. Los reactivos se agruparon en cuatro categorías: características del dispositivo, conectividad, aplicaciones y funcionalidad (véase figura 3). Para las características del dispositivo, los reactivos asociados alcanzan porcentajes moderados ( 75\%) para atributos como peso y tamaño, duración de la batería y resolución de la pantalla. Los atributos mas sancionados ( 55\%) fueron la capacidad de procesar múltiples tareas, la calidad de cámara de video y el manejo de pantalla táctil. Respecto a la conectividad, el atributo mejor evaluado (79\%) fue la capacidad para acceder a Internet, mientras que el peor evaluado se relaciona con el manejo de email (63\%). Con relación al manejo de aplicaciones, los smartphones tuvieron una valoración moderada ( $80 \%)$. Llama la atención el atributo que tiene que ver con la organización de los archivos por su baja valoración (54\%). La facilidad de manejo fue el aspecto mejor evaluado en cuanto a la funcionalidad del dispositivo (75\%) mientras que la capacidad de ver los textos en la pantalla del dispositivo en espacios exteriores fue valorada con (63\%).

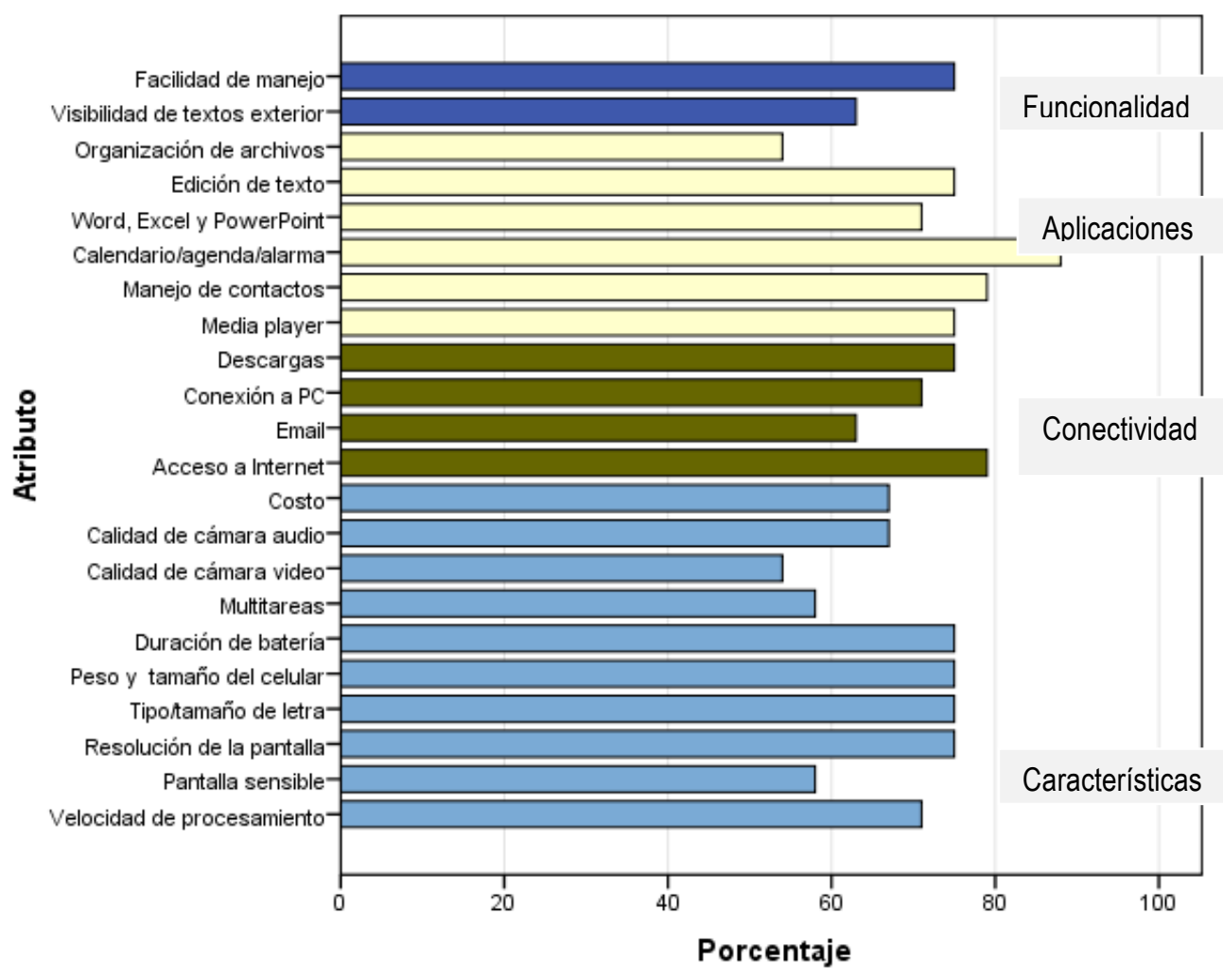

Fig. 3. Descripción de los smartphones utilizados 


\subsection{Del Espacio de Colaboración Móvil}

Como parte de los resultados, a continuación se muestran las principales opciones del ECM, visto a través de la pantalla de uno de los smpartphones utilizados en la investigación. La figura 4 muestra la pantalla inicial. Ahí se describe el propósito del ECM, a quienes va dirigido y se presentan las primeras indicaciones para acceder. Para ello, el sistema solicita el ingreso de un número celular como clave de usuario, mismo que previamente fue capturado y validado.

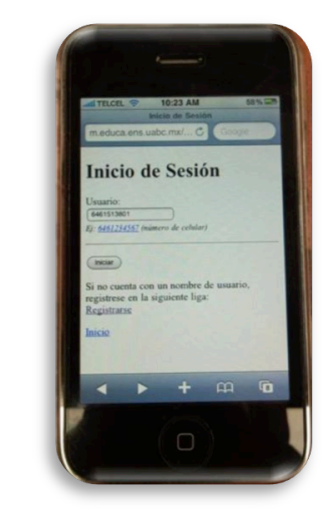

Fig. 4. Ingreso de clave de usuario

Mediante la opción PROGRESO del ECM, se proporciona la descripción de la tarea o proyecto por realizar. Se incluyen las características deseables del producto, preguntas por contestar así como la fecha límite de entrega (véase fig. 5).

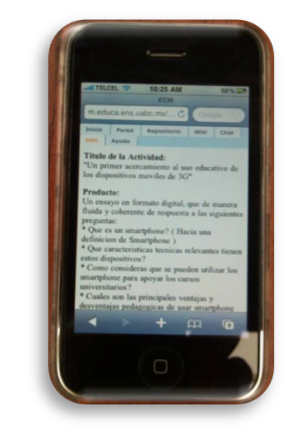

Fig. 5. Descripción de la actividad por realizar

La opción de AVISOS del ECM fue el espacio de captura y publicación de información relevante relacionada con el desarrollo de la actividad. Se les sugirió a los participantes que los mensajes fueran breves para su adecuada visualización en la pantalla reducida de los dispositivos portátiles. El orden de aparición de los mensajes fue del más reciente al más antiguo (véase fig. $6)$. 


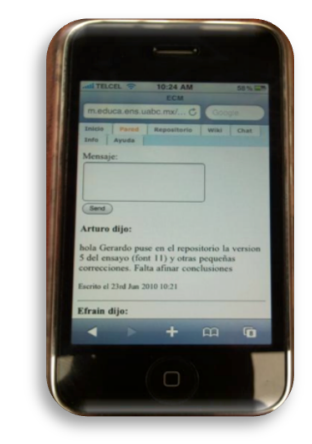

Fig. 6. Opción AVISOS

Mediante la opción de REPOSITORIO, los participantes dispusieron de espacio físico en el servidor para alojar información (archivos) de apoyo al desarrollo de la actividad. La información que colocaban quedaba compartida para todos los miembros de grupo (véase fig. 7).

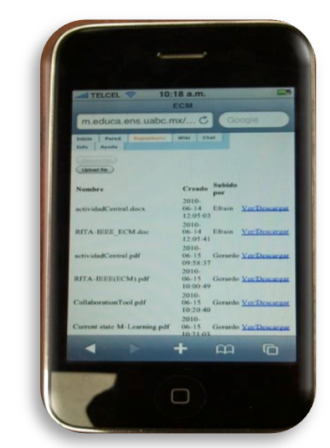

Fig. 7. Opción REPOSITORIO

A través del CHAT, los participantes del grupo intercambiaron comentarios de forma síncrona. Las comunicaciones que se establecieron fueron de participante a participante así como participación grupal (véase fig. 8).

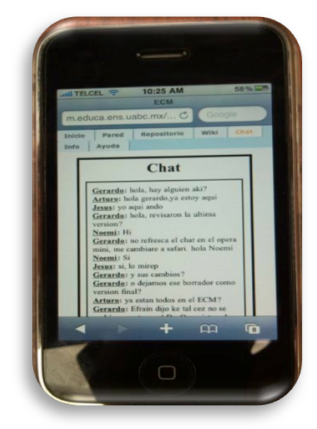

Fig. 8. Opción CHAT 
La opción denominada CONVERGENCIA, es una Wiki donde a través de una página web, un documento podía editarse, de forma simultánea, por los distintos miembros del grupo. Esto permitió la construcción colaborativa de documentos y los acuerdos entre los participantes (véase fig. 9).

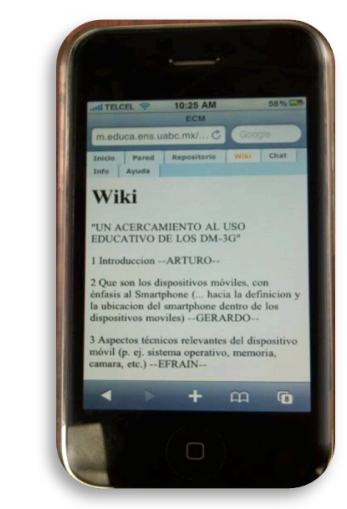

Fig. 9. Opción CONVERGENCIA/ WIKI

Además de las opciones mostradas, el espacio de colaboración ECM tuvo las opciones de CONTACTO, donde se colocaron los perfiles e identificación necesaria para localizar a los miembros de cada grupo y de AYUDA, que de manera breve mostraba la información para localizar a la persona responsable del soporte técnico del servidor y mostraba algunas respuestas a preguntas más frecuentes.

\subsection{De la m.Actividad}

Durante diez días, el grupo de investigación desarrolló de forma colaborativa un ensayo que dio cuenta de las bondades y limitaciones de los smartphones como herramienta pedagógica. Como producto, se incorporó al repositorio del ECM un documento en formato Word, con una extensión de 11 páginas. La temática y organización de dicho ensayo se muestran en la tabla 2. 
"Un primer acercamiento al uso educativo de los smartphones"

1. Introducción. Presenta una semblanza de la evolución tecnológica de los dispositivos móviles.

2. Tipos de dispositivos móviles. Se parte de la definición de Smartphone y se incluye una clasificación de dichos dispositivos con base en sus características.

\section{Aspectos técnicos relevantes de los smartphones. Se} describen las características técnicas distintivas de dichos dispositivos.

4. El proceso m-educativo. Se presenta una visión del proceso educativo cuando se considera al smartphone como herramienta pedagógica. Se incluyen aspectos asociados al docente, a los estudiantes y a los contenidos.

\section{Ventajas y desventajas del uso de smartphones en} educación superior. Incluye una serie de reflexiones acerca de las bondades y limitaciones del uso de estos dispositivos en cursos de educación superior.

6. Conclusiones. Se presenta una reflexión del potencial de uso educativo de la tecnología portátil, de los retos a corto plazo y algunas consideraciones pedagógicas.

Tabla 2. Organización del ensayo de la m.Actividad

\section{DISCUSIÓN}

Este apartado se organiza de forma similar al capítulo de resultados. Primeramente se presentan algunas interpretaciones y análisis acerca de los smarphones utilizados. Después se señalan algunas ventajas y desventajas del Espacio de Colaboración Móvil. Como tercer punto, se analiza la implementación de la actividad o trabajo grupal. Finalmente, se plantean algunas reflexiones acerca del potencial pedagógico de los dispositivos portátiles.

Con relación a las bondades de la utilización de smartphones para la realización de la actividad académica, se señalaron su portabilidad, conectividad, personalización y lo amigable de su uso. Se logró la conexión en todo momento y lugar entre los miembros del grupo. También señalaron algunos problemas, entre los que destacan la reducida visualización de la pantalla en espacios exteriores y la limitada cantidad de texto que se puede mostrar sobre la pantalla a un tiempo dado. Un aspecto importante a considerar es la forma de ingresar información desde un smartphone. Especial atención requieren el tipo de teclado y la forma de administrar las 
funciones de un ratón (mouse). Por otro lado, los programas o aplicaciones para smartphones disponibles en Internet, pueden o no ejecutarse o tener comportamientos impredecibles debido a problemas de compatibilidad derivada de los distintos sistemas operativos disponibles para tales dispositivos. Las aplicaciones mayormente utilizadas estuvieron relacionadas con agenda, alarma o calendario, manejo de contactos y comunicación. Un punto señalado por todos los participantes fue el alto costo de conexión que manejan las compañías proveedoras de telefonía celular.

Por su parte, El Espacio de Colaboración Móvil requirió alojarse en un servidor web con configuración especial para el manejo e identificación de los smartphones. Para el desarrollo programático se utilizaron algunos módulos del dominio público y el resto fue programación propia. EI ECM se accedió de manera eficiente y estable. La opción de pared de texto para los avisos fue particularmente útil para publicar mensajes cortos y mantener un registro histórico de los mensajes enviados. Así, cualquier miembro del grupo fácilmente daba seguimiento al status de la actividad y a los acuerdos logrados. Por su parte, la opción chat mostró un funcionamiento deficiente. En gran medida por la dificultad de hilar una comunicación y lograr acuerdos cuando interactúan seis participantes simultáneamente. Aunado a lo anterior, fue difícil el manejo histórico del texto en la reducida pantalla. Se recomienda conformar grupos más pequeños, quizá con un máximo de tres o cuatro participantes y considerar algún mecanismo moderador para las participaciones. En cuanto al repositorio, se destaca la importancia de disponer de un espacio de almacenamiento compartido. Dicha opción permitió depositar archivos de utilidad para el grupo. Por su parte, la opción convergencia recurre a una Wiki para la construcción colaborativa de documentos, especialmente en cuanto a la organización de la tarea. Una de las principales bondades del ECM, señalada por los participantes, fue la capacidad de registrar la evolución de la actividad colaborativa. Se podían tener registros de los avances y eventualmente aplicar acciones remediales. A manera de sugerencia, los participantes opinaron la necesidad de mejorar el diseño del ECM para que todas las opciones puedan consultarse desde el dispositivo portátil.

Acerca de la actividad colaborativa realizada con apoyo del ECM y acceso desde smartphones se destaca lo siguiente. El trabajo colaborativo requirió de un mínimo de conocimientos y habilidades por parte de los participantes. Vista la actividad como un proceso, se identificaron algunas etapas como: motivación, socialización, de acuerdos, flujos de información y síntesis. Si bien se abordaron adecuadamente las etapas antes mencionadas, una situación que se presentó fue durante la elaboración de los cuerpos de información, ya que los participantes mencionaron la dificultad de construir el documento desde el smartphone por lo que recurrieron a sus equipos de cómputo de escritorio o laptop. Por su parte, el líder del proyecto puntualizó que su labor requirió de un gran esfuerzo para lograr que cada participante entregara su parte en tiempo y forma. La labor de moderación requiere de habilidades colaborativas que generalmente no se tienen o se desconocen, por lo que se sugiere capacitar a los participantes en torno al trabajo colaborativo y sus roles. Adicionalmente, la coordinación de los esfuerzos por parte del líder de proyecto genera un costo por envío de los mensajes de texto (SMS) y transferencia de datos, lo que dificultó la coordinación del trabajo y de las reuniones virtuales. 
A manera de cierre, se puntualiza el enorme potencial pedagógico que se puede tener con los smartphones. Indudablemente se requiere que el propietario conozca sus principales capacidades y funciones. Diversos estudios (Naismith, Lonsdale, Vavoula y Sharples, 2009) han señalado que el contacto frecuente de los estudiantes con la tecnología portátil propicia el desarrollo de habilidades para operar dichos dispositivos, especialmente en cuanto a la búsqueda y consulta de información de grandes bancos y bibliotecas digitales ubicadas en la Web. De acuerdo con Sharples, Taylor y Vavoula (2009), los dispositivos portátiles propician la integración hacia redes sociales. La posibilidad de comunicación inmediata le proporciona al propietario de dichos dispositivos la percepción de integración a una red social y/o de pertenencia a una comunidad. Generalmente, las redes sociales conforman puntos virtuales de encuentro para los estudiantes con intereses y responsabilidades académicas comunes. Las redes sociales apoyan las funciones de intercambio de información, colaboración y comunicación entre los miembros de la red. Uno de los principales retos que se tiene en nuestra comunidad universitaria es la concepción misma de los smartphones como herramienta pedagógica y la coexistencia armónica de los intereses pedagógicos y recreativos de los estudiantes.

\section{REFERENCIAS}

ÁLVAREZ, D. y SCHACTHER, M. E. (2006). "El teléfono móvil: una herramienta eficaz para el aprendizaje activo," Current Developments in Technology- Assisted Education, p. 23+, Disponible: http://www.formatex.org/micte2006/pdf/23-30-A.pdf

BERGER, S., MOHR, R. NÖSEKABEL, H. K. \& SCHÄFER, J. (2003). "Mobile Collaboration Tool for University Education," Proceedings of the Twelfth IEEE International Workshops on Enabling Technologies: Infrastructure for Collaborative Enterprises (WETICE'03), pp. 77-78, 2003. [En línea].

Disponible:

http://csdl.computer.org/comp/proceedings/wetice/2003/1963/00/19630077.pdf

BROWN, T. H. (2005). "Beyond constructivism: Exploring future learning paradigms", Education Today, issue 2, Aries Publishing Company, Thames, New Zealand

BROWN, J. (2009). "Exploring Mobile Learning: Part One of the mLearning Series," Consultado el 30 de octubre de 2010, en: http://na.blackberry.com/eng/solutions/industry/education/WP_JudyBrown_Part1Long_HighR es_MobileLearning.pdf

CORBEIL, J. R. y VALDES-CORBEIL, M. E. (2007). "Are you ready for Mobile Learning?" EDUCAUSE Review, Vol. 30, No. 2, May/June 2007. Disponible en: http://www.educause.edu/EDUCAUSE+Quarterly/EDUCAUSEQuarterlyMagazineVolum/AreYou ReadyforMobileLearning/157455

FIFE, E. y PEREIRA, F. (2005). "Adoption of Mobile Data Services: Towards a Framework for Sector Analysis", M. Pagani (ed), Mobile and Wireless Systems Beyond 3G: Managing New Business Opportunities. Hershey, Pa, USA: IRM Press. 
KANTEL, E., TOVAR, G. y SERRANO, A. (2010).“Diseño de un entorno colaborativo móvil para apoyo al aprendizaje a través de dispositivos móviles de tercera generación". Revista Iberoamericana de Tecnologías del Aprendizaje (IEEE RITA), 5, 4, pp. 146-151.

KOOLE, M. (2009). A Model for Framing Mobile Learning. En: Ally, M. (Ed.). Mobile Learning: Transforming the Delivery of Education \& Training. Edmonton, AB: AU Press, Athabasca University.

KOOLE, M. \& ALLY, M. (2009) "A Model for Framing Mobile Learning," Mobile Learning Transforming the Delivery of Education and Training Athabasca University. Disponible: http://www.aupress.ca/books/120155/ebook/992_Mohamed_Ally_2009-MobileLearning.pdf

KUKULSKA-HULME, A. (junio, 2007). "Mobile Usability in Educational Contexts: What have we learnt?," en The International Review of Research in Open and Distance Learning, 8, (2). Disponible en: http://www.irrodl.org/index.php/irrodl/article/view/356/879

MANSFIELD, I. (2011). Mobile Broadband Subscription to Hit One Billion in 2011, Consultado el 7 de febrero de 2011, en: http://www.itu.int/ITUD/ict/newslog/Mobile+Broadband+Subscriptions+To+Hit+One+Billion+In+2011.aspx

NAISMITH, L., LONSDALE, P., VAVOULA, G. y SHARPLES, M. (2009). "Literature Review in Mobile Technologies and Learning", Report 11. FutureLab Series 2009. Disponible en: http://archive.futurelab.org.uk/resources/documents/lit_reviews/Mobile_Review.pdf

PRENSKY, M. (2001). "Digital natives, Digital immigrants. On the Horizon", MCB University Press, Vol. 9, No. 5, Disponible en: http://www. marcprensky.com/

SERRANO-SANTOYO, A. \& ORGANISTA-SANDOVAL, J. (2010). Implications of 4 G connectivity related to m-learning contexts. Journal of the Research Center for Educational Technology, 6, 1, 129-135. ISSN 1948-075X

SHARPLES, M. (2005). "Learning As Conversation:Transforming Education in the Mobile Age" en Conference on Seeing, Understanding, Learning in the Mobile Age, 2005. Disponible: http://www.eee.bham.ac.uk/sharplem/Papers/Theory\%20of\%20learning\%20Budapest.pdf

SHARPLES, M., TAYLOR, J. y VAVOULA, G. (2009)."A Theory of learning for the Mobile Age". Disponible en:http://www.Isri.nottingham.ac.uk/msh/Papers/Theory of Mobile Learning.pdf

TRAXLER, J. (2007). "Defining, discussing, and evaluating mobile learning: the moving finger writes and having writ...", International Review of Research in Open and Distance Learning, 8 (2), 1-8.

SHARPLES, M., TAYLOR, J. y VAVOULA, G. (2009). "A Theory of learning for the Mobile Age". Consultado el 15 de junio de 2010 en:

http://www.Isri.nottingham.ac.uk/msh/Papers/Theory\%20of\%20Mobile\%20Learning.pdf 


\section{Para citar este artículo:}

ORGANISTA, J.; SERRANO, A. (2011) «Implementación de una actividad educativa en modo colaborativo con apoyo de smarthphones: una experiencia universitaria» [artículo en línea]. EDUTEC, Revista Electrónica de Tecnología Educativa. Núm. 36 / Junio 2011. [Fecha de consulta: $\mathrm{dd} / \mathrm{mm} / \mathrm{aa}]$.

http://edutec.rediris.es/revelec2/revelec36

ISSN 1135-9250 
Anexo A.

\section{CUESTIONARIO DE EVALUACION FUNCIONAL DE SMARTPHONES}

Instrucciones. La presente encuesta pretende estimar la funcionalidad de un teléfono celular. Por favor, para cada enunciado marca el círculo que corresponda con tu apreciación de acuerdo a la escala proporcionada.

Nombre:

Equipo a evaluar:

\section{No Pésimo/a Regular Bueno/a Excelente} ATRIBUTO O CAPACIDAD DEL CELULAR aplica

$\begin{array}{llllll}\text { La velocidad de procesamiento ante } & 0 & 0 & 0 & 0 & 0 \\ \text { requerimientos del usuario } & & & 0 & 0 & 0\end{array}$

(touchscreen)

$\begin{array}{llllll}\text { La resolución de la pantalla (pixels) } & 0 & 0 & 0 & 0 & 0 \\ \text { La identificación de iconos y textos en } & 0 & 0 & 0 & 0 & 0\end{array}$

espacios exteriores

$\begin{array}{llllll}\text { El tamaño (Font) de la letra en pantalla } & 0 & 0 & 0 & 0 & 0 \\ \text { La conectividad a Internet } & 0 & 0 & 0 & 0 & 0 \\ \text { La navegación en Internet } & 0 & 0 & 0 & 0 & 0 \\ \text { El envío y recepción de mensajes por } & 0 & 0 & 0 & 0 & 0\end{array}$

email

$\begin{array}{llllll}\text { La conexión con una PC para intercambiar } & \mathrm{O} & \mathrm{O} & \mathrm{O} & \mathrm{O} & \mathrm{O}\end{array}$ información

$\begin{array}{llllll}\text { Capacidad para descargar y ejecutar } & 0 & 0 & 0 & 0 & 0\end{array}$ programas de Internet

$\begin{array}{llllll}\text { La calidad de las fotos } & 0 & 0 & 0 & 0 & 0 \\ \text { La calidad en la grabación de videos } & 0 & 0 & 0 & 0 & 0\end{array}$

(control luz; resolución)

$\begin{array}{llllll}\text { La calidad y volumen de la bocina } & 0 & 0 & 0 & 0 & 0 \\ \text { La calidad en la reproducción (play) de } & 0 & 0 & 0 & 0 & 0\end{array}$

videos y música

$\begin{array}{llllll}\text { El manejo de contactos (directorio de } & 0 & 0 & 0 & 0 & 0\end{array}$

$\begin{array}{llllll}\text { El manejo de programas de tiempo: } & \mathrm{O} & \mathrm{O} & \mathrm{O} & \mathrm{O} & \mathrm{O}\end{array}$

calendario/agenda/alarma.

$\begin{array}{llllll}\text { El manejo de programas como: Word, } & \text { O } & \text { O } & \text { O } & \text { O } & \text { O }\end{array}$

Excel y PowerPoint

$\begin{array}{llllll}\text { Capacidad de manejar dos o más } & 0 & 0 & 0 & 0 & 0\end{array}$

aplicaciones al mismo tiempo 


\begin{tabular}{|llllll|}
\hline La eficiencia en la captura de texto & 0 & 0 & 0 & 0 & 0 \\
El manejo y organización de archivos & 0 & 0 & 0 & 0 & 0 \\
$\begin{array}{l}\text { Facilidad y amabilidad de manejo del } \\
\text { celular }\end{array}$ & 0 & 0 & 0 & 0 & 0 \\
$\begin{array}{l}\text { El peso y tamaño del celular } \\
\text { La duración de la carga de la batería }\end{array}$ & 0 & 0 & 0 & 0 & 0 \\
$\begin{array}{l}\text { El costo del equipo de acuerdo a lo que } \\
\text { ofrece }\end{array}$ & 0 & 0 & 0 & 0 & 0 \\
\hline
\end{tabular}

